\title{
La traduction face aux Romans de Mélusine de Jean d'Arras et Couldrette
}

\author{
Jean-Jacques Vincensini
}

\begin{abstract}
Une tradition proprement française de la traduction conduirait à ne pas traduire, mais à s'approprier le texte-source, à l'embellir. Ce non-traduire français n'épargne pas le médiéviste national. D'autant qu'il rêve de gommer ainsi l'écart entre la littérature médiévale et la culture contemporaine. La thèse du "même au même" fonde ce point de vue dans le champ de la langue et celui de la traduction. Conséquence? Pendant longtemps, la traduction des oeuvres médiévales a semblé mineure aux yeux des médiévistes. Mais qu'en disent les publications bilingues (et "intralinguales") qui fleurissent depuis plusieurs années? Quelles stratégies et quels stéréotypes fondent leur pratique? Pour ce qui concerne les deux romans de Mélusine, tenant à faire de l'altérité une vertu, Matthew Morris et moi-même avons souhaité traduire en gardant la "bonne distance", aussi bien vis à vis du respect de la lettre du texte médiéval que du penchant ethno-centriste à en faire une œuvre contemporaine. Nos deux traductions, par ailleurs, se sont appuyées sur l'idée selon laquelle une traduction est une transformation maîtrisée par le souci de servir le sens de l'œuvre. Cet article montre alors comment ces principes généraux ont inspiré, de façon différente, nos traductions particulières, celle de la prose de Jean d'Arras et celle des vers de Couldrette. Ainsi a-t-on espéré faire de nos traductions des aventures de Mélusine et de ses fils autre chose qu'un simple exercice technique: une activité linguistique qui engage des choix esthétiques et culturels fondamentaux et évite le regrettable non-traduire français.
\end{abstract}

\section{Pu}

Ublié en 20i2, le Dernier ouvrage d'Antoine Berman, Jacques Amyot, traducteur français, s'interroge sur la "persistante occultation du rôle fondateur de la traduction en France". Le livre de Berman s'ouvre sur la traduction en français d'une citation éclairante de Friedrich Schleiermacher : "La plupart des peuples modernes effrayés par les difficultés de la traduction véritable, se contentent généralement de l'imitation et de la paraphrase. Qui prétendra affirmer qu'on ait jamais traduit en français 
quoi que ce soit des langues antiques et des langues germaniques" (BERMAN 2012, 16). ${ }^{1}$

Cette rude charge vise à montrer qu'il existerait une tradition proprement française de l'acte de traduire. Une tradition qui, si l'on en croit Schleiermacher et Goethe, reviendrait au fond à ne pas traduire, mais à s'approprier le texte-source, à l'embellir et à balancer alors, depuis Oresme et Amyot, entre "la Charybde de la belle infidèle et la Scylla de littéralité illisible" (Corbellari 2009, 147). Ce non-traduire français n'épargne pas le médiéviste national. En effet, comme l'affirme, Stéphane Marcotte dans sa "Typologie des intraduisibles de l'ancien français": "Les problèmes posés ne diffèrent pas fondamentalement selon que l'on traduit une langue ou un état de langue [c'est-à-dire dans une autre langue] et ceux que je soulèverai maintenant pour l'ancien français et le français moderne [soit la traduction intralinguale]" (Marcotte 2009, 161).

\section{Traduction et mémoire culturelle.}

Il convient donc de regarder maintenant avec plus de précisions les caractéristiques de la tâche de traducteur propre au médiéviste. Qu'on le veuille ou non, cette tâche s'inscrit tout d'abord dans une vision spécifique de la culture occidentale. En effet, tout jugement sur la traductibilité renvoie nécessairement à une réflexion sur notre mémoire culturelle. ${ }^{2}$ Chez le médiéviste, le plus souvent, ce lien relève de la continuité. Suturé dans une vision totalisante, aussi bien culturelle et esthétique, l'écart entre la littérature médiévale, glorieuse antécédence, et la culture contemporaine, disparaît. La thèse du "même au même" illustre ce point de vue dans le champ

1. Berman cite sa traduction, Des différentes méthodes du traduire et autres textes (datant de 1999) d'une conférence tenue par SCHLEIMACHER en1813.

2. Sur ce point Steiner estime que "tout ne peut être traduit aujourd'hui. Certains contextes ont disparu, des faisceaux de références qui, dans le passé, permettaient d'interpréter un texte maintenant opaque" $(1978,344)$. Une telle affirmation ne peut laisser le médiéviste indifférent : concerne-t-elle la traduction des textes médiévaux en français moderne ? Se pourrait-il que le Moyen Âge nous paraisse aujourd'hui étranger ? et il faudrait alors questionner cette étrangeté. Il conviendra de revenir sur ce problème, celui de notre rapport au Moyen Âge, à sa culture et à sa langue, et par conséquent à la raison pour laquelle nous souhaitons traduire ces textes. 
de la langue. Il se règle sur les réponses que l'on peut apporter aux questions posées par Corbellari (2009, 147): “jusqu’à quel point doit-on considérer l'ancien français [- et le Moyen français ! - ] comme une langue différente du français moderne ?" ou Galderisi: "quand est-ce que du même on passe à l'autre et qui déciderait d'un tel passage ? les gardiens d'une orthodoxie linguistique, que l'altérité linguistique naissante rend de plus en plus minoritaires, ou la multitude des nouveaux illettrés ?" $(2015,10)$.

Ces interrogations sont lourdes de conséquences dans le domaine de la traduction. Je renvoie à la prise de position de Michel Zink, "Du même au même. Traduire et récrire". Transposer un texte de l'état ancien de la langue à son état actuel, n'est pas vraiment traduire, car cette transposition ne serait pas "confrontation de deux langues différentes et passage de l'une à l'autre" (2000, 283). Dans ces conditions, le translateur ne peut parvenir qu'à une traduction "consternante" précisément parce qu'est niée la continuité de la langue.

Bref, la traduction est inutile, ou plus, les œuvres médiévales sont intraduisibles. L'intraduisible devient une valeur. En un mot, en un slogan: $\mathrm{Ne}$ pas traduire!

Il est donc clair que la conception continuiste fonde la très française méfiance à l'égard de la traduction-trahison et on comprend l'affirmation de Corinne Füg-Pierreville dans sa publication "Editer, traduire ou adapter les textes médiévaux" qui rappelle, en 2008, ce qu'affirmait Schleiermacher en 1813: "Pendant longtemps, cette pratique a paru mineure aux yeux des spécialistes, y compris des médiévistes, qui partaient du principe que le lecteur éclairé devait être capable de lire l'ancien français dans le texte. (. . .). Il devient donc urgent que cette activité gagne enfin ses lettres de noblesse, car le temps où l'élite pouvait accéder aux œuvres du Moyen Âge sans recourir au prisme déformant de la traduction est, hélas, bien révolu" $(2009,11)$.

\section{Traduire l'ancien français. Quelles stratégies? Quels stéréotypes?}

Avançons un peu. L'effort éditorial de ces dernières décennies, notamment les collections Lettres Gothiques et Champion classique ont bouleversé notre approche des textes médiévaux, "en systématisant l'accès direct aux œuvres originales et en proposant des traductions qui se veulent en phase avec le lecteur d'aujourd'hui" (Corbellari 2009, 159). Toujours dans le but de mettre au jour les caractéristiques de la traduction de textes médiévaux, je vais considérer maintenant quelques lignes de force des politiques 
de traduction qui inspirent ces traductions contemporaines destinées au grand public. Pour les Journées d'études des 4 et 5 septembre 2014 organisées à Poitiers ${ }^{3}$ et intitulées "De la traduction médiévale à la seconde main moderne. Théories, pratiques et impasses de la translation contemporaine", j'ai regardé trente sept traductions venues des collections Lettres Gothiques ; Bouquins, chez Robert Laffont ; La Pléiade, chez Gallimard ; GF de Flammarion ; les Traductions des Classiques du Moyen Âge (Champion) et Champion Classiques "série Moyen Âge". Je rappelle ici quelques résultats particulièrement nets de ce précédent travail.

1. Dans ce corpus de 37 textes, 36 traduisent ; 1 seule œuvre ne propose donc pas de traduction. L'argument "il ne faut pas traduire" semble ne pas avoir convaincu les 36 autres. 15 traductions légitiment leurs règles, bien moins que la moitié. Vingt et une traductions, donc, passent sous silence les choix qui les ont dirigées, y compris quand le travail du médiéviste se bornait seulement à traduire en regard d'un texte édité par un collègue antérieur.

2. Trois arguments composent le noyau minimal des justifications explicitées.

Premier de ces arguments, la "fidélité" déférente envers l'original. Cette fidélité peut-on la préciser ? Là, les avis divergent. Certains tiennent à rester fidèles à l'intelligence du texte, d'autres font le choix de la prose moderne plutôt que celui du vers pour garantir une meilleurs adéquation avec l'esprit du texte. Dans les remarquables et fouillées 26 pages de sa "Note sur la traduction", André Eskenazi, lui, soutient la nécessité de partir du sens du texte. Eracle "ne se propose pas seulement la mission de divertir le public en toute sécurité : il prétend lui transmettre un message". La pratique de traduction devra s'inspirer de "son souci de l'efficacité [qui] le conduit à bannir tous les effets qui risqueraient de distraire l'attention et de la faire se relâcher" (2002, 50-51).

Second argument, la fidélité est comme une planète centrale et constante autour de laquelle tournent des choix syntaxiques et lexicaux (je ne les évoquerai que très hâtivement) sur lesquels tous s'accordent:

a. Le souci de respecter la fluidité mélodique du texte justifie le mélange des temps, l'alternance du passé et du présent qui permet de varier le ton et le rythme du récit.

b. Plus délicat, et plus central pour moi, le débat sur la traduction des récits en vers. En 1992, Charles Mela offrait en regard du Chevalier de la Charrette de Chrétien de Troyes une traduc-

3. Dans le cadre d'une "action de rechecherche collaborative" commune au CESCM de Poitiers et du CESR de Tours. 
tion qui correspondait "ligne à ligne au texte du manuscrit" $(1992,33)$. Les rimes n'y manquaient pas. ${ }^{4}$ Pour Claris et Laris, Corinne Füg-Pierreville, déclare au contraire: "Nous avons naturellement choisi de traduire l'octosyllabe, mètre narratif, par la prose". Parallèlement, les traducteurs de Partonopeu de Blois défendent le choix de "la prose pour la version moderne plutôt que celui d'une traduction en vers [choix qui] garantira sans doute à notre adaptation une plus grande adéquation avec l'esprit du texte et une meilleure fluidité pour le lecteur d'aujourd'hui'" (O. Collet et P.-M. Joris, 58).

3. La fidélité est une arme paradoxale. D'un côté, le traducteur déclare s'effacer pour des raisons morales: "nous nous sommes efforcé de rendre, avec autant de probité que possible, le texte que nous avions établi"; mais, de l'autre, le même ne trouve pas "inconvenant d'adopter tel ou tel équivalent hardi" (Gros 2009, 1582). Ce paradoxe a des conséquences sur la tendance à faire de nombre de traductions actuelles des décalques des œuvres médiévales dans une langue artificielle de traduction (langue inexistante ?) que Berman nomme le "clerquois".

\section{Traduire les deux romans de Mélusine.}

Conscients de ses questionnements et de ces enjeux, nous avons défendu, Matthew Morris et moi-même, un principe fondateur: faire de l'altérité une vertu et, en conséquence, traduire en gardant la "bonne distance", aussi bien vis à vis du respect de la lettre du texte médiéval que du penchant ethno-centriste à en faire une ouvre contemporaine.

Nous avons d'abord fait le choix de traduire. En regard des chacune des deux narrations, celle de Jean d'Arras et celle de Couldrette, est donc présentée sa traduction intégrale. Ce vœu semble peut-être regrettable à ceux qui refusent de faire passer la "niveleuse de la traduction", selon la jolie expression de May Plouzeau (2001, 243), sur le texte-source et aux défenseurs de la conception du "même au même". Le lecteur du XXIe siècle pourrait, disent-ils, au prix d'un peu de bienveillance, aller à la découverte de cette langue du Moyen Âge flamboyant aux allures à première vue si familières. Eclairé par les notes historiques et philologiques, l'original devrait le satisfaire, évitant ainsi le soupçon qui plane sur le résultat d'une traduction,

4. Comme "En peu de temps je suis tombé / par son fait de si haut en bas" qui traduit An po d'ore m'a abessié / Voirement de si haut si bas. (v. 6476-77). 
souvent douteux et mutilant. Mais la connaissance, plus ou moins exacte, de l'origine historique ou culturelle d'une œuvre littéraire, de sa tradition manuscrite et des traits propres à son expression, donne-t-elle accès à la signification, niant ainsi l'intérêt de sa traduction?

Une raison complémentaire plaide en faveur de la traduction des aventures des Lusignan, elle tient à la fameuse altérité du Moyen Âge et à l'incessante fascination qu'elle exerce de nos jours. Ce temps que nous aspirons à connaître paraît proche: des pans entiers de sa langue, de sa culture, de son art, de sa foi semblent accessibles. N'est-ce pas ce sentiment de familiarité qui s'impose en ouvrant une page de Mélusine ? En réalité, le Moyen Âge est inexorablement loin de nous. Laltérité de ses œuvres est un fait. Divers traits incontestables, d'ordre culturel, historique et linguistique, creusent l'étrangeté qui nous écarte de l'époque de nos deux romans, d'autant plus quand son accès prend l'allure d'une séduisante proximité. Bref, il fallait traduire.

Je voudrais voir maintenant comment ces principes généraux ont inspiré, de façon différente, nos traductions particulières, celle de la prose de Jean d'Arras et celle des vers de Couldrette.

Conséquence directe du principe que je viens de souligner, nous avons admis, en premier lieu, qu'une traduction est une transformation, maîtrisée par le souci de servir le sens de l'œuvre. ${ }^{5}$ Quel sens ?

Dans le prolongement des observations avancées dans mon introduction de la Mélusine de Jean d'Arras (2003), je me suis adossé à l'idée selon laquelle ce roman exprime un véritable mythe qui, comme il se doit, appelle une multiplicité d'écritures génériques. En second lieu, j’ai tenu compte de l'évidence: Jean d'Arras offre à la lecture une masse imposante, aux ramifications narratives complexes témoignant souvent d'un indiscutable attrait pour l'emphase (voir Cerquiglini 1981, 13); ce récit use et abuse, par ailleurs, des termes réalistes et techniques. Si tant est qu'il soit compréhensible, ce récit est-il alors lisible, ce qui est loin d'être identique ? Ces constats de départ ont engagé les options suivantes.

Premièrement, ils ont conduit à privilégier le lexique concret et figuratif, quitte à conserver tels quels certains termes C'est ainsi que, sans excès d'originalité, j'ai traduit l'expression en la vertu (de ta chamberiere Nature,

5. La conservation de la division en chapitre et des paragraphes tels que les indiquent les rubriques, les dessins colorés et les lettres capitales peintes de notre manuscrit s'inscrit dans cette même perspective. Ces caractéristiques ne sont pas seulement formelles, ce sont des procédés esthétiques chargés de significations. 
8va) par "dans les mains (de la nature, ta servante)" sans ignorer que le substantif vertu est généralement porteur d'un sens modal et abstrait ("pouvoir", "puissance", "disposition"). C'est le même souci qui a incité à traduire le vocabulaire abstrait (comme preud'homme). Pour les phrases sentencieuses et les proverbes, je n'ai pas cherché les équivalences modernes. La terrible menace de Geoffroy: je (. . .) mettray tout en feu et en flambe (119vb), n'est pas rendue par le tentant "je mettrai tout à feu et à sang", mais par: "j'y mettrai le feu, tout flambera". J'ai conservé les proverbes ayant des énoncés modernes quasi-identiques, renforçant alors l'allure stéréotypée de ces propositions en introduisant parfois "dit-on", absent de l'original. Par exemple "Ne dit-on pas : À peuple indigent, seigneur mendiant ?" translate Car se peuple est povre, le seigneur est mendiz $(44 v b)$.

Comme le fait le manuscrit, je me suis efforcé de conserver les mots différents qui expriment une même notion (tenir compte, notamment, du fait qu'une tour peut-être crenelee, guerlandee ou couronnee); par ailleurs, dans la mesure du possible, et sans excès de témérité, il a paru judicieux de retrouver les sonorités originales et de respecter les formes lexicales peu attestées ("décourage" pour descuerre, par exemple 96vb) en évitant la paraphrase. Conformément aux résultats de la comparaison des 37 textes évoquée plus haut, j'ai tenté de préserver la syntaxe arborescente de cette écriture, ses prolepses, ses retours incantatoires, un certain rythme (pas toujours le sien), la variation alternative des temps de la narration, les faveurs qu'elle accorde aux répétitions et aux doublets. Enfin, j'ai pris en compte cette "polylogie informe" et irrégulière du roman dont j'ai parlé à l'instant, la variété des tons propres à chaque genre: prologue et épilogue philosophiques, qu'il ne s'agit pas, avant tout, de rendre clairs. Aristote est-il clair ? Il a fallu tenir compte non seulement des caractères des épisodes usant de la prose narrative sans contours nets et sans rythme harmonieux dont a bien parlé Jens Rasmussen (1958, 43), mais des nombreux récits brefs, des divers passages didactiques (relevant du genre "Miroir des Princes") et des épisodes épiques (aventures outre-mer des fils de Mélusine) ou encore de ceux qui ressemblent à des chroniques historiques.

Je passe à la traduction de Couldrette qui, parce qu'elle est fondée - je le répète - sur les mêmes principes, paradoxalement, suit des pistes tout à fait singulières. Nous avons adopté une présentation versifiée, ce qui la distingue du travail en prose de Laurence Harf-Lancner (1993) traduisant la version établie par Eleanor Roach. C'est que, dans le prolongement des observations littéraires que Matthew et moi-même présentons dans notre introduction, nous avons voulu que la traduction de Couldrette expose la vision - très différente en cela de Jean d'Arras — du romanesque qui ins- 
pire sa narration. Le regard nostalgique du récit en vers nous a incités à traduire dans la volonté précise de faire mine, à notre tour, d'obliquer vers le vieillot. D'où les deux effets suivants.

Le premier tient à l'usage du vers, mais d'un vers libre et rythmé par une mesure qui peut être différente de celle du poème de Couldrette. Cette "distance" a conduit à ne pas sauvegarder systématiquement l'octosyllabe, ni à maintenir méthodiquement les rimes de notre modèle, sauf quand le français moderne s'y prêtait. Par exemple, "Je mettrai mon corps, ma pensée et mon cœur / À rehausser votre honneur" (v. 662-63) ou "De son corps, l'âme s'en est allée./ Qu’à Dieu elle soit recommandée !" (v. 2331-32).

Seconde conséquence : le recours volontaire à un vocabulaire évoquant une époque révolue ("Dieu te pardonne toutes les fautes / Que tu as commises à mon encontre", par exemple; v. 4081-82) et à des tours syntaxiques un peu surannés, comme : "À la mêlée, grande est la clameur : / Lusignan ! lancent-ils à pleins poumons" (v. 1944-45) ou encore "Arrivent à la tête de nombreux Poitevins / Qui sont nourris de très bons vins" (v. 2417-18; "Se nourrir de vins" ne se dit — malheureusement ? - plus ...).

Il est temps de conclure. Certes, comme l'écrit Daniel O'Sullivan dans la Préface de notre édition de Couldrette, notre "monde où la volonté de connaissance et de puissance tend à débusquer les traces de l'imaginaire a besoin d'histoires comme celle de Mélusine" (2009, x). Mais comment accéder à cette œuvre écrite au xve siècle, comment goûter ses aventures si la langue qui les raconte reste incomprise ? Pour la plus grande partie du public contemporain, la traduction est le medium nécessaire pour lire et aimer cette légende qui permet de "découvrir les vestiges de cultures éloignées que n'effrayaient ni monstres ni merveilles" (2009, x). Traduire les œuvres médiévales, comme Mélusine, n'est donc pas seulement un exercice technique, c'est une activité linguistique majeure qui engage des choix esthétiques et culturels fondamentaux.

Au cours du temps, dynamique et heureux, passé à penser, faire et parfaire notre traduction du poème de Couldrette, Matthew m'a donné l'occasion, de ne pas sombrer dans le dangereux non-traduire français et de féconder la vie de la langue française qu'il aimait tant et qu'il parlait si "bellement". Je l'en remercie très sincèrement.

\section{Ouvrages cités}

Berman, Antoine. 2012. Jacques Amyot, traducteur français. L'extrême contemporain. Paris: Belin. 
Cerquiglini, Bernard. 1981. La Parole médiévale. Paris: éd. de Minuit, 1981.

Chrétien de Troyes. 1992. Le Chevalier de la Charrette ou le Roman de Lancelot. Edité et traduit par Charles MéLA. Lettres Gothiques. Paris: LGF.

Corbellari, Alain. 2009. "Traduire l'ancien français en français moderne: petit historique d'une quête inachevable". In Éditer, traduire ou adapter les textes médiévaux, Actes du Colloque international, 11-12 décembre 2008, Lyon, Université Jean Moulin, 147-160. Dirigé par Corinne Füg-Pierreville. Lyon: Publication du CEDIC.

Coudrette. 1993. Le Roman de Mélusine. Traduit par Laurence Harf-Lancner. GF. Paris: Flammarion.

Couldrette. 2009. Mélusine (Roman de Parthenay ou Roman de Lusignan). Edité et traduit par Matthew W. Morris et Jean-Jacques Vincensini. The Edwin Mellen Press, Lewiston, Queenston, Lampeter.

Collet, Olivier et Pierre-Marie Jorris, eds. et trad. 2005. Partonopeu de Blois. Lettres Gothiques. Paris: LGF.

Füg-Pierreville, Corinne. 2007, éd et trad. Claris et Laris. Traduction des Classiques français du Moyen Âge 157. Paris: Champion.

Galderisi, Claudio. 2015. "Un truchement me faut quérir ... Peut-on traduire pour qui ne connaît pas le français médiéval ?" In De l'ancien français au français moderne. Théories, pratiques et impasses de la traduction intralinguale, 7-32. Études réunies par Claudio Galderisi et Jean-Jacques Vincensini. Bibliothèque de Transmédie. Turnhout: Brepols.

Gautier d'Arras, 2002. Éracle. Edité par Guy Raynaud de Lage et traduit par André EskénAzi. Traduction des Classiques français du Moyen Âge 54. Paris: Champion.

Gros, Gérard, éd. 2009. La Quête du saint Graal. In Le Livre du Graal. Bibliothèque de La Pléiade. Paris: Gallimard.

Jean d'Arras. 2003. Mélusine ou La Noble Histoire de Lusignan. Roman du XIVe siècle. Edité et traduit par Jean-Jacques Vincensini. Lettres Gothiques. Paris: LGF.

Marcotte, Stéphane, 2009. "Typologie des intraduisibles de l'ancien français". In Editer, traduire ou adapter les textes médiévaux, Actes du Colloque international, 11-12 décembre 2008, Lyon, Université Jean Moulin, 161-96. Dirigé par Corinne Füg-Pierreville. Lyon: Publication du CEDIC.

Plouzeau, May. 2001. "Compte rendu de Perceforest 2e p. t. I, éd. critique de G. Roussineau, 1999” Romania $119: 242-71$.

Rasmussen, Jens. 1958. La Prose française du XVe siècle. Copenhague: E. Munksgaard.

Steiner, George. 1978. Après Babel. Une poétique du dire et de la traduction. Traduit par Lucienne Lotringer. Paris: Albin Michel.

ZIN K, Michel. 2000. "Du même au même. Traduire et récrire". In Perspectives médiévales. Suppl. au n ${ }^{\circ}$ 26. Actes du Colloque "Translation médiévale", Mulhouse 11-12 mai 2000, 283-90. Dirigé par Claudio Galderisi et Gilbert Salmon. 